\title{
MicroRNA-429 acts as a tumor suppressor in colorectal cancer by targeting high mobility group box 3
}

\author{
XIANGYANG TIAN ${ }^{1}$, JIANLAN CHANG ${ }^{1}$, NINGNING ZHANG ${ }^{1}$, SHOUXIN WU $^{2}$, HUIMIN LIU ${ }^{2}$ and JUNYAN YU ${ }^{1}$ \\ ${ }^{1}$ Department of Oncology, Heping Hospital, Changzhi Medical College, Changzhi, Shanxi 046000; \\ ${ }^{2}$ Biotecan Medical Diagnostics Co., Ltd., Zhangjiang Center for Translational Medicine, Shanghai 201203, P.R. China
}

Received February 28, 2020; Accepted December 10, 2020

DOI: $10.3892 / \mathrm{ol} .2021 .12511$

\begin{abstract}
Colorectal cancer (CRC) is one of the most common solid tumors worldwide and has an extremely poor prognosis. MicroRNA-429 (miR-429) has been reported to participate in the progression of CRC. However, the pathological mechanisms require further investigation. The aim of the present study was to investigate the association between miR-429 and high mobility group box 3 (HMGB3) in CRC and the associated mechanism. The mRNA expression levels of miR-429 and HMGB3 in 65 paired CRC and adjacent tissues were examined by reverse transcription-quantitative PCR. Furthermore, a dual-luciferase reporter assay was performed to identify the association between miR-429 and HMGB3. Finally, the effects of miR-429 and HMGB3 on the proliferation and apoptosis of CRC cells were detected. As a result, it was identified that miR-429 expression was downregulated and HMGB3 expression was upregulated in CRC tissues compared with in adjacent non-cancer tissues, and the expression levels of miR-429 were negatively associated with those of HMGB3. Notably, HMGB3 was demonstrated to be a direct target of miR-429 by dual-luciferase reporter assay. Furthermore, transfection with a miR-429 mimic significantly inhibited HMGB3 expression and led to decreased proliferation and increased apoptosis of CRC cells. On the other hand, transient overexpression of HMGB3 partially inhibited the antitumor effects of miR-429. To the best of our knowledge, the present study demonstrated for the first time that miR-429 regulated the proliferation and apoptosis of CRC cells via HMGB3, suggesting a specific tumor suppressive function of the miR-429/HMGB3 signaling pathway in CRC.
\end{abstract}

Correspondence to: Dr Junyan Yu, Department of Oncology, Heping Hospital, Changzhi Medical College, 110 South Yan'an Road, Changzhi, Shanxi 046000, P.R. China

E-mail: hanbing398@163.com

Dr Huimin Liu, Biotecan Medical Diagnostics Co., Ltd., Zhangjiang Center for Translational Medicine, 180 Zhangheng Road, Shanghai 201203, P.R. China

E-mail: hmliu@biotecan.com

Key words: microRNA-429, high mobility group box 3, colorectal cancer, tumor suppressive function

\section{Introduction}

Colorectal cancer (CRC) is one of the most commonly diagnosed malignancies, and in recent years, the incidence rate of CRC has shown an increasing tendency; it was estimated that there were $>1.2$ million newly diagnosed patients with CRC and $\sim 600,000$ patients died due to CRC in 2019, worldwide (1). However, the pathogenesis of CRC remains unclear, and current standard treatments for CRC remain a combination of surgery, chemotherapy and radiotherapy; in numerous cases, none of the current treatment methods achieve the desired therapeutic effects, and the prognosis of patients with CRC remains poor (1-3). Therefore, there is an urgent need to identify novel therapeutic targets and develop new methods for the treatment of CRC with improved therapeutic efficacy.

In recent years, the roles of microRNA (miRNAs or miRs) in carcinogenesis have been investigated in numerous studies $(4,5)$. miRNAs are endogenous non-coding mRNAs with a length of 18-25 nucleotides, which can bind to the 3'-untranslated region (UTR) of mRNAs and suppress the expression of their target proteins at the post-transcriptional level (6). In the field of CRC, miRNAs have been reported to participate in the occurrence and development of the disease, either as tumor suppressors or promoters $(7,8)$. For example, it has been observed that miR-503 can promote the migration and invasion of CRC cells via targeting programmed cell death 4 (PDCD4) (9). By contrast, miR-185 has been reported to enhance the radiosensitivity of CRC cells (10). These results suggest that miRNAs are promising therapeutic targets for CRC. miR-429 is a member of the miR-200 family, and we previously reported the tumor suppressive role of miR-429 in colon cancer cell lines (11). High mobility group box 3 (HMGB3) has been reported to be an oncogene (12-15). However, to the best of our knowledge, the molecular mechanisms underlying the association between miR-429 and HMGB3 in CRC are yet to be elucidated.

The present study aimed to identify the association between miR-429 and HMGB3 in CRC and the underlying mechanism.

\section{Materials and methods}

Patients and samples. A total of 65 patients with primary CRC who underwent curative resection at Heping Hospital (Changzhi, 
China) were recruited between May 2016 and July 2017. Patients who did not receive preoperative neoadjuvant therapy were included in the study. The main characteristics of these 65 patients are presented in Table I. Fresh frozen tumor tissue and adjacent normal tissue samples ( $>5 \mathrm{~cm}$ from the margin of the tumors) were collected from each patient. The experiments were performed with the understanding and written informed consent of each patient, and the investigation was performed in accordance with the Declaration of Helsinki. The present study was approved by the Medical Ethics Committee of Heping Hospital, Changzhi Medical College.

Cell culture and transfection. The human CRC LOVO cell line was obtained from the Cell Bank of Type Culture Collection of the Chinese Academy of Sciences. LOVO cells were cultured in high-glucose Dulbecco's modified Eagle's medium (DMEM; Gibco; Thermo Fisher Scientific, Inc.) supplemented with $10 \%$ fetal bovine serum (Cytiva) and $1 \%$ penicillin/streptomycin (Thermo Fisher Scientific, Inc.), and maintained in a humidified incubator at $37^{\circ} \mathrm{C}$ with $5 \% \mathrm{CO}_{2}$. DNA constructs, including miR-429 mimics (5'-UGCCAAAAU GGUCUGUCAUAAU-3'), miR-429 mimic negative control (NC; scrambled sequence; 5'-UUCUCCGAACGUGUCACG UTT-3') and HMGB3 overexpressing plasmid (empty vector was used as NC), were designed by Shanghai GenePharma Co., Ltd. miR-429 mimics and miR-429 mimic NC sequences were ligated into a pGCMV vector. The cells were transfected with Lipofectamine ${ }^{\circledR} 3000$ reagent (Invitrogen; Thermo Fisher Scientific, Inc.), using plasmid DNA (1-2 $\mu \mathrm{g})$. The cells were transfected with miR-429 mimics or miR-429 mimics in combination with HMGB3 overexpressing plasmids. The transfected cells were incubated at $37^{\circ} \mathrm{C}$ with $5 \% \mathrm{CO}_{2}$, and the subsequent experiments were performed $48 \mathrm{~h}$ after transfection. The successful transfections of miR-429 mimics and HMGB3 overexpressing constructs in LOVO cells are shown in Fig. S1. Researchers performing the aforementioned assays were blinded to sample information, and all experiments were repeated with at least three independent culture preparations.

Cell proliferation assay. A Cell Counting Kit-8 (CCK-8) cell proliferation assay (Sigma-Aldrich; Merck KGaA) was performed to detect the viability of cells at 12,24 and $48 \mathrm{~h}$ post-transfection. LOVO cells were seeded onto 96-well plates at $1 \times 10^{3}$ cells/well and incubated with $100 \mu 1$ DMEM containing $10 \mu \mathrm{l} \mathrm{CCK}-8$ solution at $37^{\circ} \mathrm{C}$ for $2 \mathrm{~h}$ at each time point. The optical density value at $450 \mathrm{~nm}$ was then examined to determine the proliferative ability of the cells.

Apoptosis assay. The apoptosis of LOVO cells was determined using an Annexin V apoptosis detection kit (Nanjing KeyGen Biotech Co., Ltd.). Briefly, cells were stained with Annexin V-FITC and propidium iodide (PI) for 10-15 min at room temperature, and the apoptosis of cells was examined using a BD FACSCalibur flow cytometer (BD Biosciences). The experimental results were analyzed using FlowJo version 10.4 (FlowJo LLC). The apoptosis between different groups was compared and illustrated in results at $48 \mathrm{~h}$ post-transfection.

Bioinformatics analysis. TargetScan (http://www.targetScan. org/vert_72) predicts biological targets of miRNAs by searching for the presence of conserved 8-mer, 7-mer and 6-mer sites that match the seed region of each miRNA. The human HMGB3 gene was searched on the website, with miR-429 as the microRNA.

Dual-luciferase reporter assay. 293 cells (Cell Bank of Type Culture Collection of the Chinese Academy of Sciences) were used for the dual-luciferase reporter assay. The cells were cultured in DMEM supplemented with $10 \%$ fetal calf serum (Cytiva). pGL3 plasmids (Promega Corporation) containing the wild-type (WT) 3'-UTR of HMGB3 or mutated (MT) 3'-UTR of HMGB3 were constructed. WT or MT plasmids were co-transfected with miR-429 mimics or NC using Lipofectamine 3000 reagent. Cells were harvested $24 \mathrm{~h}$ later, and luciferase activity, which was compared with Renilla luciferase activity and was determined using a dual luciferase assay system (Promega Corporation) according to the manufacturer's protocol.

Reverse transcription-quantitative PCR (RT-qPCR). Total RNA was extracted from tissue and cell samples using TRIzol ${ }^{\circledR}$ reagent (Invitrogen; Thermo Fisher Scientific, Inc.) according to the manufacturer's protocol. RT was performed using PrimeScript $^{\mathrm{TM}}$ RT Master mix (Perfect Real Time) kit (Takara Bio, Inc.) according to the manufacturer's protocol. qPCR was subsequently performed using the Mir- ${ }^{\mathrm{TM}}$ miRNA qRT-PCR SYBR $^{\circledast}$ kit (Takara Bio, Inc.) for miR-429 mRNA expression (with U6 used as the internal reference gene) and SYBR Green qPCR Master mix (Takara Bio, Inc.) for HMGB3 mRNA expression (with GAPDH used as the internal reference gene). The following thermocycling conditions were used: Initial denaturation at $95^{\circ} \mathrm{C}$ for $15 \mathrm{sec}$ followed by 40 cycles at $95^{\circ} \mathrm{C}$ for $5 \mathrm{sec}$ and $60^{\circ} \mathrm{C}$ for $30 \mathrm{sec}$. All reactions were performed in duplicates, and the comparative $2^{-\Delta \Delta \mathrm{Cq}}$ method was used for comparisons between samples unless otherwise stated (16). Primers are listed in Table SI.

Western blot analysis. Tissues and cell samples were harvested using radioimmunoprecipitation lysis buffer (Beyotime Institute of Biotechnology), and the protein concentration was determined using a BCA Protein assay kit (Beyotime Institute of Biotechnology). Total protein was then separated using $10 \%$ SDS-PAGE, and transferred to a PVDF membrane. Subsequently the membrane was blocked with 5\% skimmed milk in PBS for $4 \mathrm{~h}$. Next, the membrane was incubated with specific primary antibodies for $3 \mathrm{~h}$ at room temperature. After being washed with PBS three times, for 5 min each time, the membrane was incubated with the appropriate secondary antibody for $2 \mathrm{~h}$ at room temperature. After washing with PBS three times, for $5 \mathrm{~min}$ each time, an enhanced chemiluminescence western blotting kit (Cytiva) was used to detect the immune complexes on the membrane. The following antibodies were used: Anti-HMGB3 (1:1,000; cat. no. PA5-68709; Thermo Fisher Scientific, Inc.), anti-GAPDH $(1: 2,000$; cat. no. ab8227; Abcam) and HRP goat anti-rabbit (1:2,000; cat. no. ab7090; Abcam).

Statistical analysis. Statistical analysis was performed using GraphPad Prism (version 6.01; GraphPad Software, Inc.). Data are presented as the mean \pm standard deviation. The differences between two groups were compared by paired or 
Table I. Main characteristics of the patients included in the study $(n=65)$.

\begin{tabular}{ll}
\hline Characteristic & Number of patients \\
\hline
\end{tabular}

\section{Han nationality}

Yes

No

Family history

Yes
No

Smoking

Yes

No

Drinking

Yes 0

No

Sex

Male

Female

Age, years

$>50$

$\leq 50$

Age range, years

Median age, years

Tumor site

Colon

Rectum

Tumor size, $\mathrm{cm}$

$\geq 3$

Number of lesions

1

$>1$

Not available

TNM stage

I-II

III-IV

Differentiation

Well/moderate $\quad 39$

Poor

Lymphatic metastasis

Positive

Negative

Preoperative neoadjuvant therapy

Yes

No

unpaired t-test, and the differences among multiple groups were analyzed by one-way analysis of variance with Turkey's

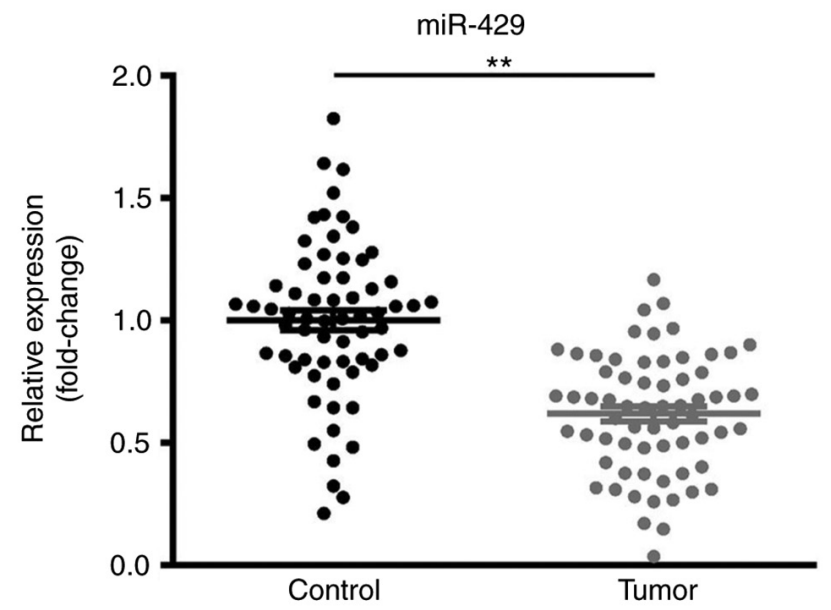

Figure 1. Relative miR-429 expression in colorectal cancer (tumor) and adjacent normal tissues (control). ${ }^{* *} \mathrm{P}<0.01$. miR-429, microRNA-429.

post-hoc test. Pearson's correlation coefficient was used to determine the correlation between miR-429 and HMGB3 expression in tumor samples. $\mathrm{P}<0.05$ was considered to indicate a statistically significant difference.

\section{Results}

Downregulation of miR-429 in CRC tissue samples. The expression levels of miR-429 in CRC and matched adjacent non-cancer tissues were examined by RT-qPCR. As presented in Fig. 1, miR-429 expression was significantly decreased in $\mathrm{CRC}$ tissues compared with in the corresponding non-cancer colorectal tissues.

Tumor suppressive function of miR-429 in LOVO cells. The tumor suppressive role of miR-429 in CRC has been reported previously (11). The present study further validated these previous findings by investigating the effects of miR-429 on the proliferation and apoptosis of LOVO cells. As a result, transient overexpression of miR-429 induced a significant decrease in the proliferation and a significant increase in the apoptosis of LOVO cells (Fig. 2A and B).

HMGB3 is a direct target of miR-429. Using the online bioinformatics tool Targetscan, HMGB3 was predicted as a direct target of miR-429 (Fig. 3A). To confirm the association between miR-429 and HMGB3, a dual-luciferase reporter assay was conducted. Notably, luciferase activity was significantly decreased in 293 cells co-transfected with the reporter containing the WT 3'-UTR of HMGB3 and miR-429 mimics, while co-transfection of the reporter containing MUT 3'-UTR of HMGB3 and miR-429 mimics did not affect luciferase activity (Fig. 3B). These results provided direct evidence that HMGB3 was a target of miR-429.

Expression levels of miR-429 and HMGB3 are negatively correlated in CRC tissue samples. To further investigate the association between miR-429 and HMGB3 in CRC, the expression levels of HMGB3 in CRC tissues and matched adjacent non-cancer tissues were examined. It was observed that 

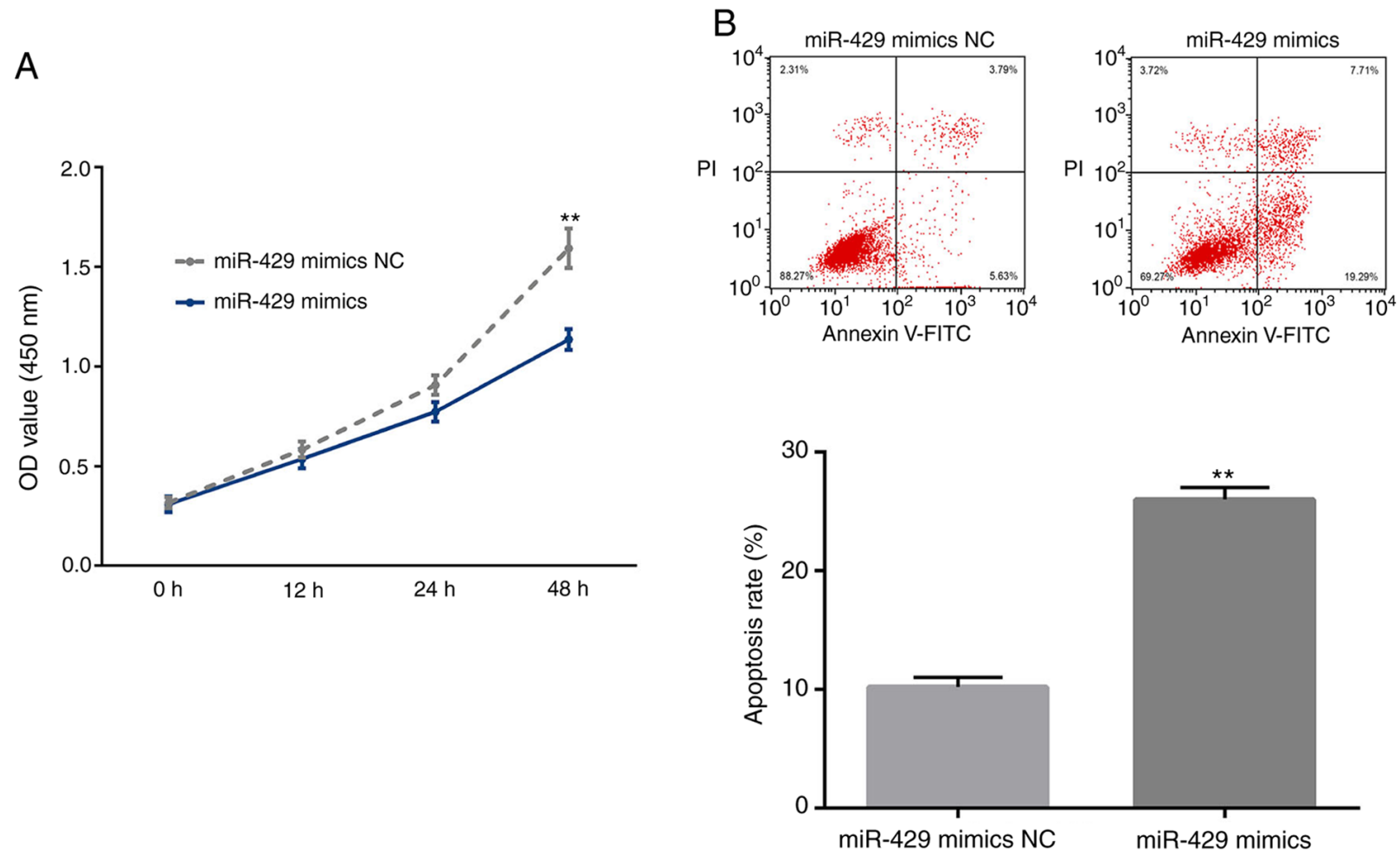

Figure 2. Effect of miR-429 on the proliferation and apoptosis of LOVO cells in vitro. (A) Effect of miR-429 on the proliferation of LOVO cells in vitro. (B) Effect of miR-429 on the apoptosis of LOVO cells in vitro. ${ }^{* *} \mathrm{P}<0.01$. miR-429, microRNA-429; NC, negative control; OD, optical density; PI, propidium iodide.

\section{A} $\begin{array}{lll}\text { Position 837-844 of HMGB3 3'UTR } & 5^{\prime} & \text {...UUGAAGUUAAAUAAACAGUAUUA... } \\ \text { hsa-miR-429 } & 3^{\prime} & \text { UGCCAAAAUGGUCUGUCAUAAU } \\ \text { Mutant position of HMGB3 3'UTR } & 5^{\prime} & \text {...UUGAAGUUAAAUAAAGACACGGA... }\end{array}$

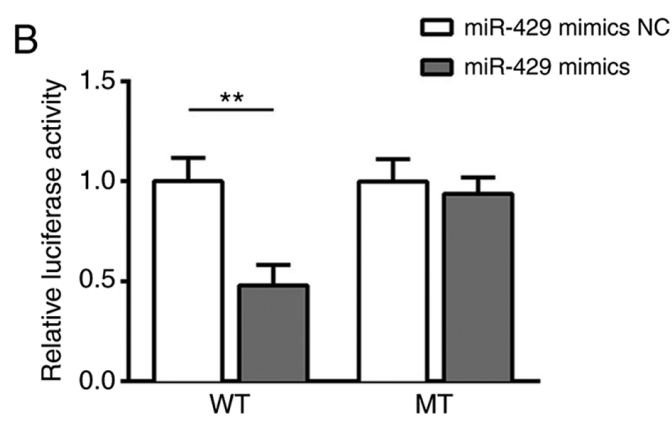

Figure 3. HMGB3 acts as a direct target of miR-429. (A) Sequence alignment of the paired 3'-UTR of miR-429 and HMGB3. (B) Results of dual-luciferase reporter assay. ${ }^{* *} \mathrm{P}<0.01$. WT, wild-type; MT, mutated; HMGB3, high mobility group box 3; UTR, untranslated region; miR-429, microRNA-429; $\mathrm{NC}$, negative control.

HMGB3 was significantly upregulated in CRC tumor tissues compared with in the adjacent tissues at both the protein and mRNA levels (Fig. 4A-C). Furthermore, the expression levels of HMGB3 were negatively correlated with those of miR-429 in CRC tissue samples (Fig. 4D).

HMGB3 is involved in the antitumor effect of miR-429. To further identify whether HMGB3 is involved in the antitumor effect of miR-429, LOVO cells were divided into the following three groups according to the transfection performed: i) Control group; ii) miR-429 mimics group; and iii) miR-429 mimics and HMGB3 overexpression plasmid group. The protein expression levels of HMGB3 in each group were determined by western blotting. It was demonstrated that transfection with miR-429 mimics significantly decreased the protein expression levels of HMGB3, which were then restored by HMGB3 overexpression (Fig. 5A). Furthermore, transient overexpression of miR-429 mimics induced a significant decrease in the proliferation and a significant increase in the apoptosis of LOVO cells, while concomitant overexpression of HMGB3 significantly rescued these tumor suppressive effects of miR-429 (Fig. 5B and C). Thus, it was demonstrated for the first time that miR-429 functioned as a tumor suppressor in CRC via targeting HMGB3.

\section{Discussion}

$\mathrm{CRC}$ is one of the most common and lethal malignancies worldwide. Therefore, there is an urgent requirement to improve the understanding of the pathological mechanisms of CRC and explore more effective methods for CRC diagnosis and treatment. miR-429 exerts a tumor suppressive function in CRC (11); however, the molecular mechanism remains unclear. The present study investigated miR-429 and its target gene HMGB3, and their underlying mechanism in the progression of CRC.

The role of miR-429 in CRC is not clearly defined. Han et al (17) observed that miR-429 expression was upregulated in CRC tissue compared with that in adjacent non-tumor 
A
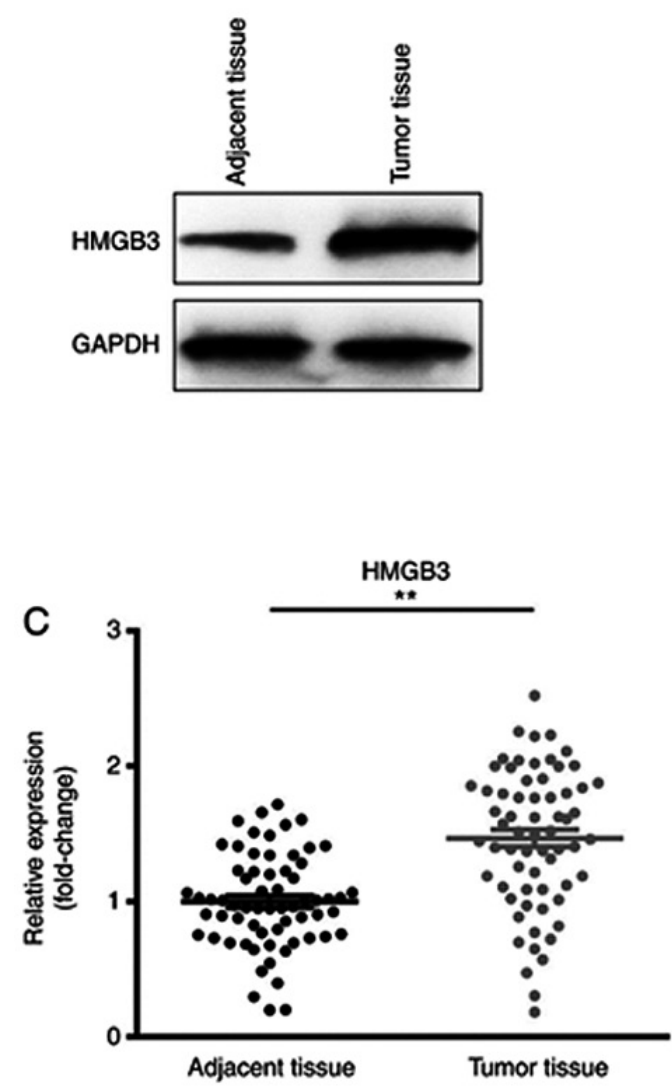

B

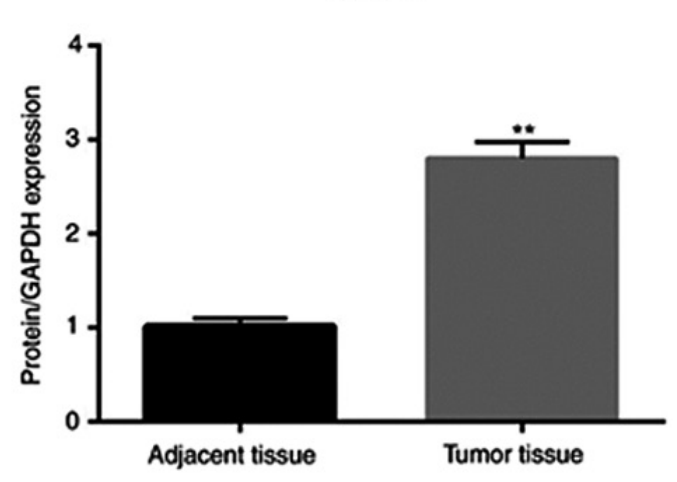

D

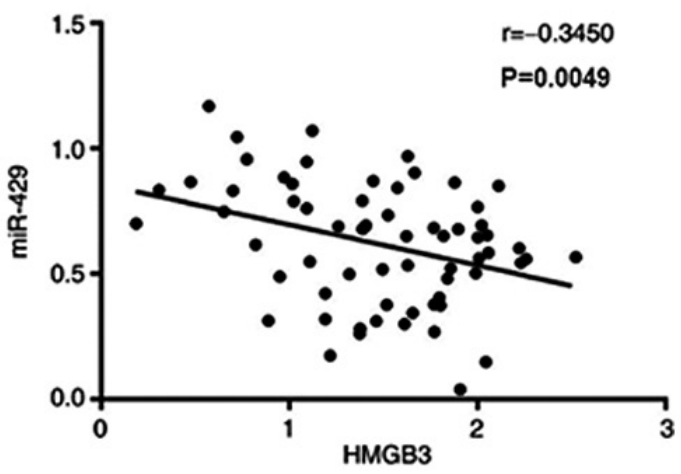

Figure 4. Roles of HMGB3 in CRC. (A) Relative HMGB3 expression in CRC tissues and adjacent tissues at the protein level. (B) Quantification of the western blot results. (C) Relative HMGB3 expression in CRC and adjacent tissues at the mRNA level. (D) Correlation between the expressions levels of miR-429 and HMGB3 in CRC tumor tissues. ${ }^{* *} \mathrm{P}<0.01$. CRC, colorectal cancer; HMGB3, high mobility group box 3; miR-429, microRNA-429.

tissue, and increased the proliferation and migration of CRC cells by targeting homeobox A5, a transcription factor of HOX families. Comparatively, Sun et al (18) suggested that miR-429 inhibits cell proliferation and invasion, and regulates epithelial-mesenchymal transition-associated marker genes by targeting Onecut 2 in CRC. In addition, we previously reported that miR-429 suppresses the migration and invasion of CRC cells by targeting p21-activated kinase 6/cofilin signaling (11). These differences may be a result of the limited sample size. The present study identified that miR-429 expression was downregulated in CRC tissues compared with that in adjacent tissues, which was consistent with the results of Sun et al (18) and our previous study (11). Furthermore, the present study demonstrated that transient overexpression of miR-429 significantly decreased the proliferation and promoted the apoptosis of CRC cells in vitro. Overall, the present results indicated the tumor suppressive function of miR-429 in CRC.

It is well-known that miRNAs exert their function through suppressing the expression of their target genes. HMGB3 belongs to the HMGB family, which consists of HMGB1, HMGB2, HMGB3 and HMGB4 (19). Increasing evidence has demonstrated that HMGB3 may act as an oncogene in different types of cancer, including non-small cell lung cancer (20), breast cancer (21), esophageal squamous cell cancer (22), gastric cancer (23) and CRC (24). Notably, the current study confirmed that HMGB3 was a target gene of miR-429 using a dual-luciferase reporter assay. Furthermore, it was identified that HMGB3 expression was upregulated in CRC tissues compared with in adjacent tissues, which was consistent with the findings of Zhang et al (24). Notably, the present study first reported the negative correlation between the expression levels of miR-429 and HMGB3 in CRC tissue samples. In addition, it was identified that transfection of LOVO cells with miR-429 mimics significantly decreased HMGB3 expression, and transient overexpression of HMGB3 blocked the antitumor effects of miR-429. Thus, miR-429 may act as a tumor suppressor in the progression of CRC via suppressing HMGB3 expression. Further studies are required to explore the upstream regulatory molecules of miR-429 in CRC progression and verify whether the miR-429/HMGB3 signaling pathway is associated with patient outcome.

Overall, the present study investigated the function of miR-429 in CRC progression. Using correlation analysis and a dual-luciferase reporter assay, it was identified that HMGB3 acted as a direct target of miR-429. In addition, it was observed that miR-429 regulated the proliferation and apoptosis of CRC cells via targeting HMGB3. How precisely the regulation occurs and/or whether miR-429 interacts with other known signaling pathways regulating CRC cell proliferation and apoptosis remains to be determined in future studies. Meanwhile, other colon cancer cell lines should be used to further verify the tumor suppressive role of the miR-429/HMGB3 signaling pathway in the future. 
A

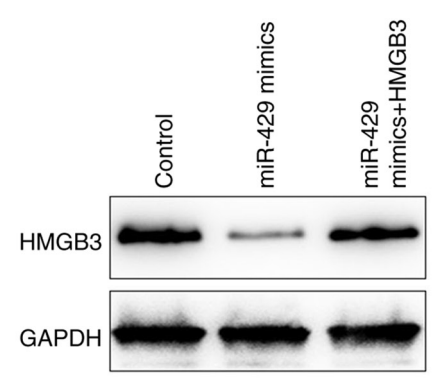

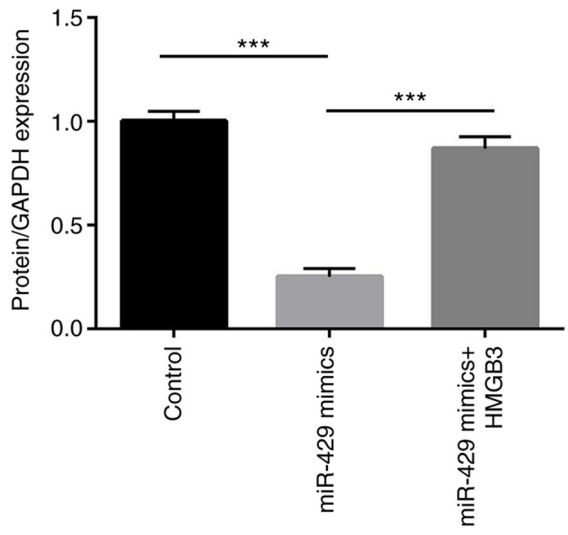

B

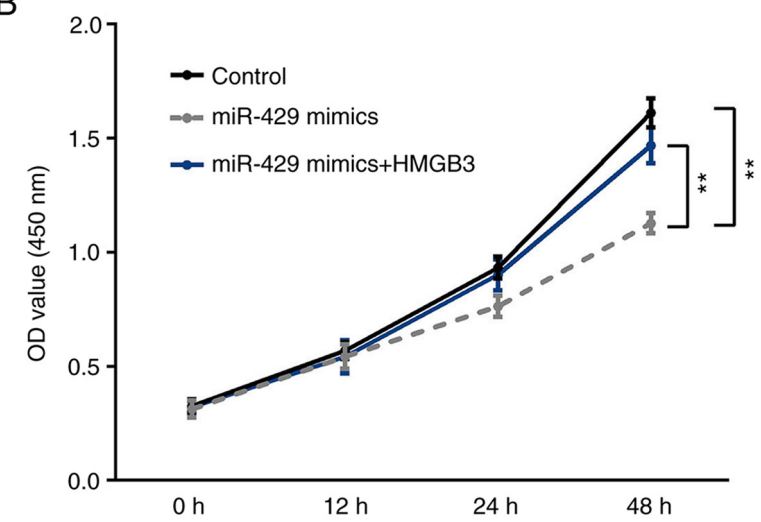

C
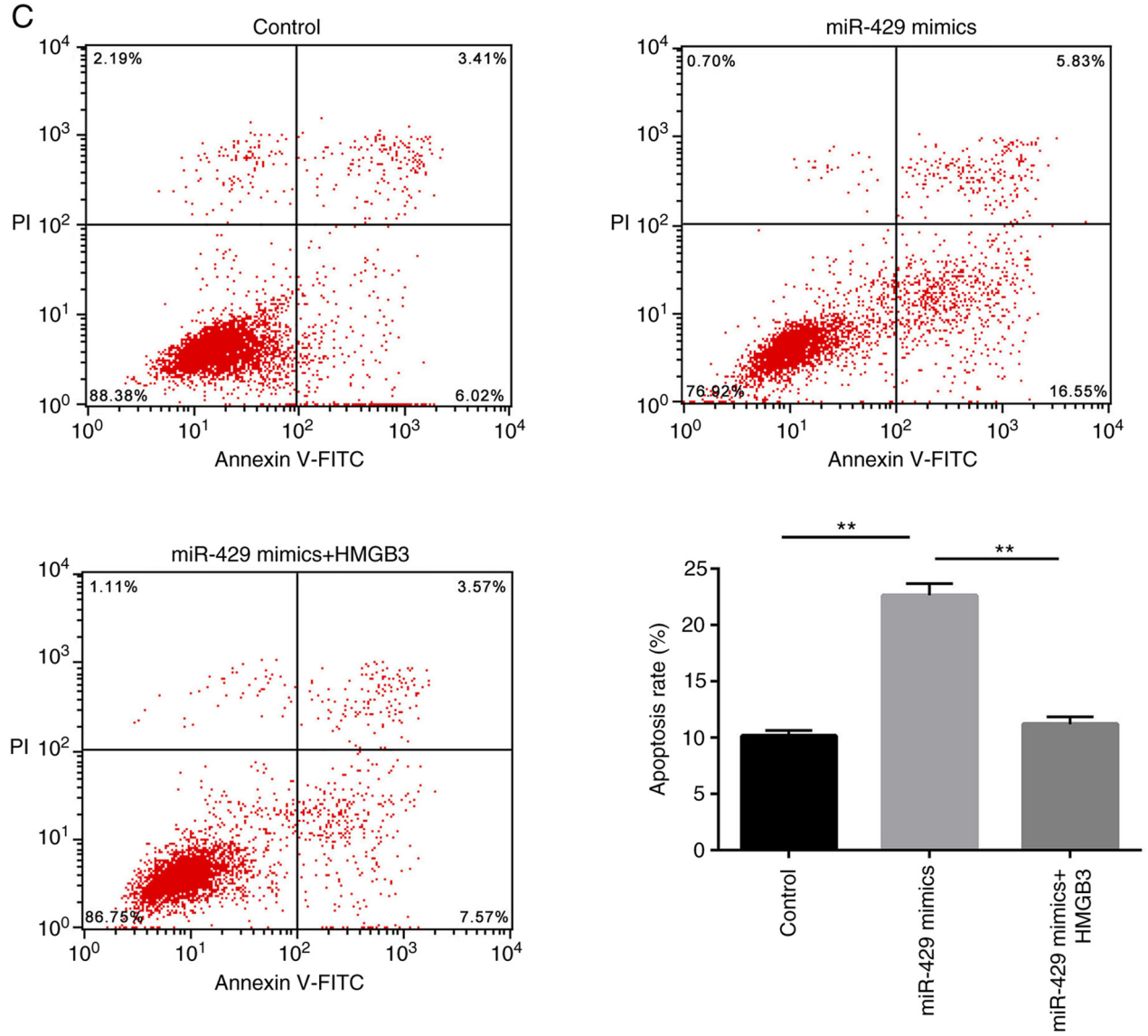

Figure 5. miR-429 regulates the proliferation and apoptosis of colorectal cancer cells via targeting HMGB3. Cells were transfected with miR-429 mimics or miR-429 mimics together with HMGB3 overexpressing plasmids. (A) HMGB3 protein expression in different groups. (B) Proliferation of LOVO cells in different groups. (C) Apoptosis of LOVO cells in different groups. ${ }^{* *} \mathrm{P}<0.01 ;{ }^{* * *} \mathrm{P}<0.001$. miR-429, miroRNA-429; HMGB3, high mobility group box 3; OD, optical density; PI, propidium iodide. 


\section{Acknowledgements}

Not applicable.

\section{Funding}

The present study was funded by the Supporting Fund for Scientific Research of Changzhi Medical College (grant no. QDZ201610).

\section{Availability of data and materials}

The datasets used and/or analyzed during the current study are available from the corresponding author on reasonable request.

\section{Authors' contributions}

JY and SW conceived the study and designed the experiments. XT performed the experiments and analyzed the data. HL conceived the study, analyzed the data and wrote the manuscript. JC and NZ collected samples and analyzed the clinical data. All authors read and approved the final manuscript. JY and SW confirm the authenticity of all the raw data. All authors read and approved the final manuscript.

\section{Ethics approval and consent to participate}

All procedures performed in studies involving human participants were in accordance with the ethical standards of the Medical Ethics Committee of Heping Hospital (Changzhi, China), and with the 1964 Declaration of Helsinki and its later amendments or comparable ethical standards. Written informed consent was obtained from all individual participants that were included in the present study.

\section{Patient consent for publication}

Not applicable.

\section{Competing interests}

The authors declare that they have no competing interests.

\section{References}

1. Gormley JA, Hegarty SM, O'Grady A, Stevenson MR Burden RE, Barrett HL, Scott CJ, Johnston JA, Wilson RH, Kay EW, et al: The role of cathepsin $\mathrm{S}$ as a marker of prognosis and predictor of chemotherapy benefit in adjuvant CRC: A pilot study. Br J Cancer 105: 1487-1494, 2011.

2. Nikolouzakis TK, Vassilopoulou L, Fragkiadaki P, Mariolis Sapsakos T, Papadakis GZ, Spandidos DA, Tsatsakis AM and Tsiaoussis J: Improving diagnosis, prognosis and prediction by using biomarkers in CRC patients (Review). Oncol Rep 39: 2455-2472, 2018.

3. Wu J, Wang F, Liu X, Zhang T, Liu F, Ge X, Mao Y and Hua D: Correlation of IDH1 and B7H3 expression with prognosis of CRC patients. Eur J Surg Oncol 44: 1254-1260, 2018.

4. Costa DF and Torchilin VP: Micelle-like nanoparticles as siRNA and miRNA carriers for cancer therapy. Biomed Microdevices 20: 59, 2018.

5. Xu B, Liu J, Xiang X, Liu S, Zhong P, Xie F, Mou T and Lai L: Expression of miRNA-143 in pancreatic cancer and its clinical significance. Cancer Biother Radiopharm 33: 373-379, 2018.
6. Chen Y, Zhao J, Luo Y, Wang Y and Jiang Y: Downregulated expression of miRNA-149 promotes apoptosis in side population cells sorted from the TSU prostate cancer cell line. Oncol Rep 36: 2587-2600, 2016.

7. Ju H, Tan JY, Cao B, Song MQ and Tian ZB: Effects of miR-223 on colorectal cancer cell proliferation and apoptosis through regulating FoxO3a/BIM. Eur Rev Med Pharmacol Sci 22: 3771-3778, 2018

8. Su M, Qin B, Liu F, Chen Y and Zhang R: miR-885-5p upregulation promotes colorectal cancer cell proliferation and migration by targeting suppressor of cytokine signaling. Oncol Lett 16: 65-72, 2018.

9. Li L, Zhang X, Yi Z, Liang X, Yin W and Li S: MiR-503 promotes the migration and invasion of colorectal cancer cells by regulating PDCD4. J BUON 23: 579-586, 2018.

10. Afshar S, Najafi R, Sedighi Pashaki A, Sharifi M, Nikzad S, Gholami MH, Khoshghadam A, Amini R, Karimi J and Saidijam M: MiR-185 enhances radiosensitivity of colorectal cancer cells by targeting IGF1R and IGF2. Biomed Pharmacother 106: 763-769, 2018

11. Tian X, Wei Z, Wang J,Liu P, Qin Y andZhong M: MicroRNA-429 inhibits the migration and invasion of colon cancer cells by targeting PAK6/cofilin signaling. Oncol Rep 34: 707-714, 2015.

12. Petit A, Ragu C, Della-Valle V, Mozziconacci MJ, Lafage-Pochitaloff M, Soler G, Schluth C, Radford I, Ottolenghi C, Bernard OA, et al: NUP98-HMGB3: A novel oncogenic fusion. Leukemia 24: 654-658, 2010.

13. Guo S, Wang Y, Gao Y, Zhang Y, Chen M, Xu M, Hu L, Jing Y, Jing F, Li C, et al: Knockdown of high mobility group-box 3 (HMGB3) expression inhibits proliferation, reduces migration, and affects chemosensitivity in gastric cancer cells. Med Sci Monit 22: 3951-3960, 2016

14. Li M, Cai Y, Zhao H, Xu Z, Sun Q, Luo M, Gu L, Meng M, Han X and Sun H: Overexpression of HMGB3 protein promotes cell proliferation, migration and is associated with poor prognosis in urinary bladder cancer patients. Tumour Biol 36: 4785-4792, 2015.

15. Yamada Y, Nishikawa R, Kato M, Okato A, Arai T, Kojima S, Yamazaki K, Naya Y, Ichikawa T and Seki N: Regulation of HMGB3 by antitumor miR-205-5p inhibits cancer cell aggressiveness and is involved in prostate cancer pathogenesis. J Hum Genet 63: 195-205, 2018.

16. Livak KJ and Schmittgen TD: Analysis of relative gene expression data using real-time quantitative PCR and the 2(-Delta Delta C(T)) method. Methods 25: 402-408, 2001.

17. Han Y, Zhao Q, Zhou J and Shi R: miR-429 mediates tumor growth and metastasis in colorectal cancer. Am J Cancer Res 7: 218-233, 2017.

18. Sun Y, Shen S, Liu X, Tang H, Wang Z, Yu Z, Li X and Wu M: MiR-429 inhibits cells growth and invasion and regulates EMT-related marker genes by targeting Onecut 2 in colorectal carcinoma. Mol Cell Biochem 390: 19-30, 2014.

19. Li L, Huang J, Ju Z, Li Q, Wang C, Qi C, Zhang Y, Hou Q, Hang S and Zhong J: Multiple promoters and targeted microRNAs direct the expressions of HMGB3 gene transcripts in dairy cattle. Anim Genet 44: 241-250, 2013.

20. Song N, Liu B, Wu JL, Zhang RF, Duan L, He WS and Zhang CM: Prognostic value of HMGB3 expression in patients with non-small cell lung cancer. Tumour Biol 34: 2599-2603, 2013.

21. Elgamal OA, Park JK, Gusev Y, Azevedo-Pouly AC, Jiang J, Roopra A and Schmittgen TD: Tumor suppressive function of mir-205 in breast cancer is linked to HMGB3 regulation. PLoS One 8: e76402, 2013.

22. Gao J, Zou Z, Gao J, Zhang H, Lin Z, Zhang Y, Luo X, Liu C, Xie J and Cai C: Increased expression of HMGB3: A novel independent prognostic marker of worse outcome in patients with esophageal squamous cell carcinoma. Int J Clin Exp Pathol 8: 345-352, 2015.

23. Gong Y, Cao Y, Song L, Zhou J, Wang C and Wu B: HMGB3 characterization in gastric cancer. Genet Mol Res 12: 6032-6039, 2013.

24. Zhang Z, Chang Y, Zhang J, Lu Y, Zheng L, Hu Y, Zhang F, Li X, Zhang $\mathrm{W}$ and Li X: HMGB3 promotes growth and migration in colorectal cancer by regulating WNT/ $\beta$-catenin pathway. PLoS One 12: e0179741, 2017. International (CC BY-NC-ND 4.0) License. 